Impfungen

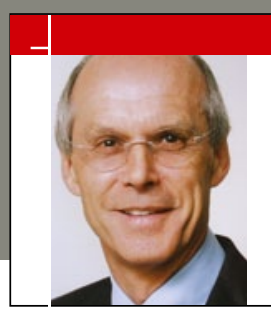

Prof. Dr. med. Hermann S. FüeßI

43-... bei Kindern

47 -... bei Erwachsenen

51 - ... bei immunsupprimierten Patienten

Leiter Somatischer Querschnittsbereich

Isar-Amper-Klinikum, Klinikum München-Ost, Haar

Impfungen im Kindes- und Erwachsenenalter

\title{
Königsweg der Prävention
}

- Impfungen gehören zweifellos zu den wirksamsten und elegantesten präventiven medizinischen Maßnahmen. Viele Geißeln der Menschheit wie z. B. die Pocken konnten durch systematische Impfprogramme weltweit eliminiert werden. Moderne Impfstoffe sind gut verträglich. Bleibende unerwünschte gravierende Arzneimittelwirkungen (UAW) werden nur in sehr seltenen Fällen beobachtet. So gesehen haben Impfungen ein ausgezeichnetes und in der Medizin fast beispielloses Nutzen-Risiko-Verhältnis.

Dennoch werden Impfungen von einem kleinen, aber harten Kern der Bevölkerung sehr kritisch betrachtet und in einer Art von fast religiösem Fanatismus abgelehnt - nicht zu reden von der weit verbreiteten Nachlässigkeit und Gleichgültigkeit. Hausärzten fällt gerade aufgrund ihrer Vertrauensposition die wichtige Aufgabe zu, Verunsicherungen und Fehlinformationen aus dem Weg zu räumen und gleichzeitig zum Impfen aufzufordern, um möglichst hohe Impfraten zu erzielen. Denn nur bei einem hohen Anteil von Geimpften kommt es zur so genannten Herden-Immunität. Darunter versteht man das Phänomen, dass sich ab einer bestimmten

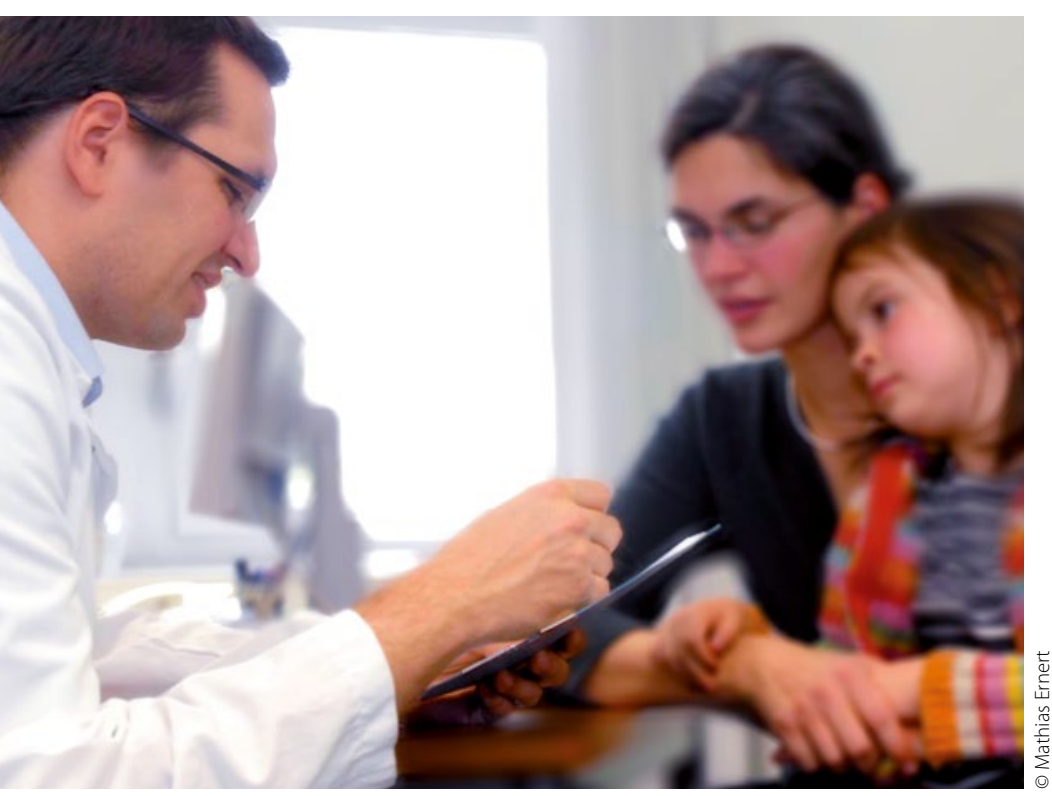

Impfrate der Bevölkerung ein Krankheitserreger nicht mehr effizient vermehren kann und deshalb ausstirbt. Das ist der (leider labile) Zustand, in dem diese Krankheit als besiegt gilt. Wie hoch diese Impfrate sein muss, hängt von der Reproduktionsrate des Erregers ab. Mit den gegenwärtigen Impfraten wird es nicht gelingen, eine Herden-Immunität gegenüber Influenza oder Varizellen zu erreichen, obwohl das theoretisch möglich wäre.

Als Initiative der Weltgesundheitsorganisation (WHO)/Regionalbüro für Europa begann daher am 21. April die Europäische Impfwoche, in der durch gezielte Kommunikationskampagnen über die Bedeutung von Impfungen informiert und dadurch die Impfraten erhöht werden sollte. Auch in Deutschland besteht noch Aufklärungsbedarf, sowohl bei den Impfungen von Kindern, als auch von Erwachsenen. So wurde von 2010 auf 2011 ein deutlicher Anstieg der Masernerkrankungen von 780 auf 1607 Fälle beobachtet. Große Impflücken gibt es auch bei den nach 1970 geborenen Erwachsenen. Gerade die neueren Empfehlungen der Ständigen Impfkommission wie die Masernimpfung für junge Erwachsene (2010) oder die 2009 ausgesproche Keuchhusten-Impfempfehlung für Erwachsene, die gemeinsam mit der nächsten fälligen Impfung gegen Tetanus und Diphtherie gegeben werden soll, sind noch nicht ausreichend bekannt.

Auch bei Erkrankungen, bei denen mittels Impfung keine lebenslange Immunität zu erzielen ist, spielt die Herden-Immunität eine wichtige Rolle, wie sich bei Pneumokokken-Infektionen gezeigt hat. Pneumonien sind die häufigste Todesursache bei Infektionskrankheiten sowohl bei Kindern, als auch bei Patienten über 65 Jahre. Durch die Einführung der 7-valenten Pneumokokken-Vakzine konnte in den USA gezeigt werden, dass sich in den Jahren 2000-2006 ca. 790000 Krankenhausaufnahmen verhindern ließen, wobei $90 \%$ dieser Reduktion auf Herden-Immunität zurückzuführen war. In Deutschland besteht keine Impfpflicht, Impfungen werden von den Gesundheitsbehörden entsprechend den STIKO-Empfehlungen nur "öffentlich empfohlen".

Ärztliche Tätigkeit ist zwar primär dem Wohl des individuellen Patienten verpflichtet, doch müssen bei Infektionskrankheiten immer auch epidemiologische Aspekte berücksichtigt werden. Wer wäre besser dafür geeignet als die Hausärzte. 